\title{
A simple technique to prevent retrograde ejaculation during assisted ejaculation
}

\author{
T C Lim MBBS FACRM,${ }^{1} \mathrm{C}$ Mallidis MSc, ${ }^{2} \mathrm{~S}$ T Hill MBBS FACRM, ${ }^{1}$ D J Skinner \\ $\mathrm{RN},{ }^{1} \mathrm{P}$ D Carter BVSc MACVSC ${ }^{3}$ D J Brown MBBS FRACP FACRM, ${ }^{1}$ \\ $\mathrm{H}$ W G Baker MD PhD FRACP ${ }^{2}$
}

${ }^{1}$ Spinal Injuries Unit, Austin Hospital, Heidelberg 3084, Australia; ${ }^{2}$ University of Melbourne Department of Obstetrics and Gynaecology and Reproductive Biology Unit, Royal Women's Hospital, 132 Grattan St, Carlton 3053, Australia and Melbourne IVF 320 Victoria Pde, East Melbourne 3002 Australia; ${ }^{3}$ Ratek Instruments, 92 Beaumont Rd, Berwick 3806, Australia.

The aim of this study was to develop a technique which would prevent
retrograde ejaculation in chronic spinal cord injured (SCI) patients undergoing
vibration and electroejaculation procedures. A balloon catheter was used to
tamponade the bladder neck in 12 patients who underwent 100 assisted
ejaculation procedures. Antegrade ejaculations were collected on all occasions
with no incidences of urine contamination and no sperm were seen in post
ejaculatory urine. Silicone catheters had minimal effects on sperm motility and
viability. All lubricant gels were found to adversely affect sperm quality and
were not used.

Keywords: assisted ejaculation; retrograde ejaculation; spinal cord injury; infertility.

\section{Introduction}

It is well recognised that semen quality in men with long standing spinal cord injury (SCI) is poor and characterised by small volumes, variable counts and in particular, poor motility. ${ }^{1,2}$ The semen may improve with repeated use of assisted ejaculation techniques-electroejaculation (EE) or vibration ejaculation (VE) - though it rarely reaches levels found in healthy, nondisabled men. ${ }^{3}$

Linsenmeyer \& Perkash $^{4}$ have listed a large number of factors that may contribute to poor semen quality including stasis of prostatic fluid, testicular hyperthermia, recurrent urinary tract infections, abnormal testicular histology, possible changes in the hypothalamic-pituitary-testicular axis, possible sperm antibodies, some medications and the type of bladder management. Other factors are also of significance, particularly retrograde ejaculation or partial retrograde ejaculation and the contamination of semen by urine during antegrade ejaculation. ${ }^{5}$
The incidence of retrograde ejaculation in chronic SCI has been reported at between $9 \%$ and $41 \% .{ }^{1-3.6}$ In these patients, sperm can be retrieved from the urine, but the procedure involves a complicated process of preparation requiring either the alteration of $\mathrm{pH}$ and osmolarity of the urine or emptying of the bladder by catheter before instilling a buffered solution or culture medium.

Warner et $a l^{6}$ have described a complex technique of modifying commercially available catheters to prevent retrograde ejaculation. An inflated balloon acted as a 'ballcock valve' blocking urine flow from the bladder into the prostatic urethra and preventing semen entering the bladder. A number 18, siliconised rubber, three-way Foley catheter had the bladder ports in the urinary lumen blocked with silicone rubber. The irrigation port was left open. Additional ports for semen to enter the urinary lumen were cut into the portion of the catheter which would reside in the prostatic urethra. It is interesting to note that 
although they reported a moderate degree of success with this particular method, in later publications the same group of workers $^{7}$ had dispensed with this catheter technique and were using post-ejaculation bladder flushes.

Rawicki \& $\mathrm{Hill}^{8}$ briefly noted the use of balloon catheter bladder tamponade in three patients to prevent retrograde ejaculation during assisted ejaculation. We use a similar method. The simple technique is described. Also, commonly used lubricants and catheters were studied to determine their effects on sperm.

\section{Materials and methods}

\section{Assisted ejaculation}

\section{Patients}

All patients volunteered for inclusion in the study. Prior to each procedure being performed, the technique was explained to the patient and a consent form completed. Twelve men with SCI (Table I) aged 21-36 (mean 31) years were treated with assisted ejaculation techniques at the Austin Hospital Spinal Injuries Unit. The procedures were performed 1-24 (mean 8) years after the injury.

\section{Electroejaculation procedure}

In all patients, a 14 French gauge one-way $100 \%$ silicone Foley catheter was introduced into the bladder by sterile technique and its balloon filled with $10 \mathrm{ml}$ of normal saline. The use of lubricants was avoided because of their harmful effects on sperm. ${ }^{9}$ The 'stickiness' of the catheter material and the flexibility of the Foley catheters made insertion difficult. This was overcome by injecting normal saline directly into the urethra with a syringe. A Twomey syringe with $50 \mathrm{ml}$ normal saline was connected to the distal end of the Foley catheter. An assistant gently infused the urethral passage with a continuous flow of normal saline as the catheter was inserted. This provided lubrication between the urethral mucosa and the catheter. Urine was drained and the balloon inflated with $10 \mathrm{ml}$ of normal saline. The assistant gently pulled on the catheter so the inflated balloon tamponaded the bladder neck (Fig 1). We did not use a weighted traction device as described by Warner et al ${ }^{6}$

A rechargeable, battery-powered electrical stimulator (Fig 2), the 'CGS Electrojector' (Ratek Industries $\mathrm{P} / \mathrm{L}$ ), provided a progressively increasing sine wave current at 20 Hertz to a maximum of 16 volts and 500

Table I Physical information, type of bladder management and assisted ejaculation procedure

\begin{tabular}{lrlll}
\hline Subjects & Month/Year of injury & Level & Bladder management & Procedure \\
\hline 1 & $4 / 68$ & C4(C) & Reflex & EE $^{\mathrm{a}}$ \\
2 & $1 / 69$ & C4(C) & Reflex & VE $^{\mathrm{a}}$ \& VE \\
3 & $6 / 77$ & C6(C) & Reflex & EE \& VE \\
4 & $11 / 81$ & C6(C) & Reflex \& EUS & EE \& VE \\
5 & $4 / 84$ & T11(C) & Straining & EE \\
6 & $12 / 85$ & T10(C) & Reflex & EE \\
7 & $12 / 86$ & T11(C) & Reflex \& EUS & EE \\
8 & $12 / 87$ & T10(C) & ICSC & EE \\
9 & $3 / 88$ & T12(I) & Reflex & EE/GA \\
10 & $7 / 88$ & L2(C) & & Reflex \& EUS \\
11 & $1 / 90$ & C6(I) & VE & \\
12 & $1 / 91$ & T8(C) & ICSC & EE \\
\hline
\end{tabular}

${ }^{a}$ Nifedine prophylaxis used.

EUS $=$ external urethral sphincterotomy.

ICSC $=$ intermittent clean self catheterisation.

$\mathrm{EE} / \mathrm{GA}=$ electroejaculation performed under general anaesthesia. 


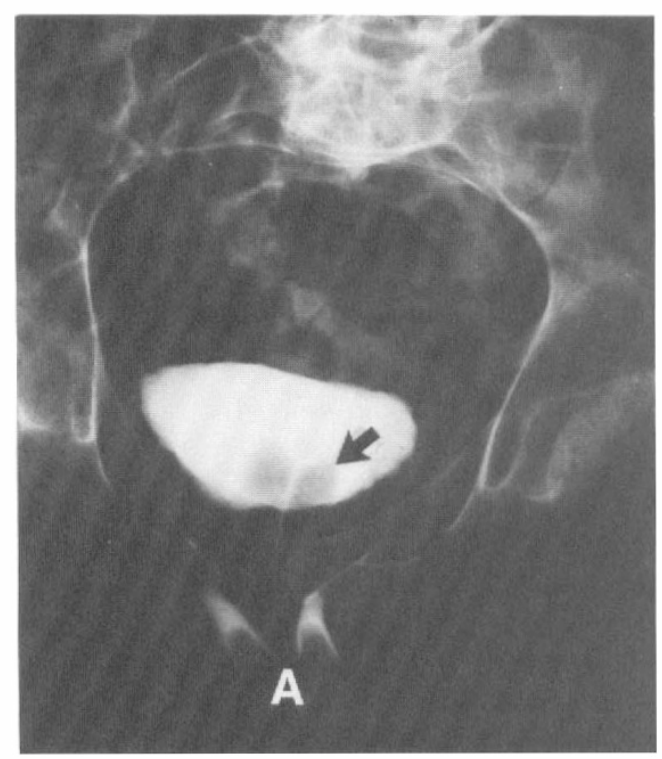

Figure 1 Foley catheter tamponading the bladder neck. The arrow indicates the balloon of the catheter opposed to the bladder neck. 'A' indicates incidental bilateral penile implants.

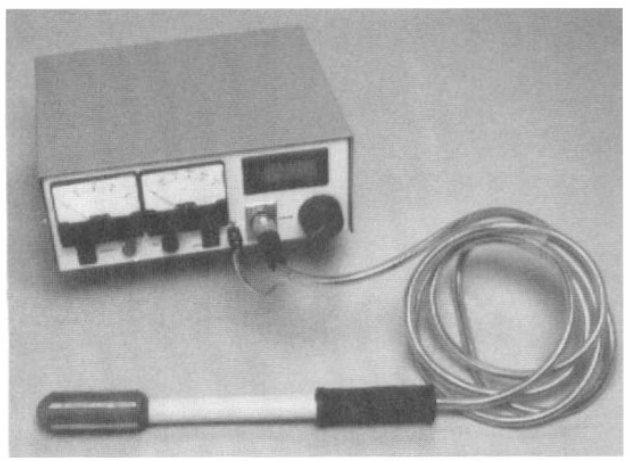

Figure 2 CGS Electrojector and probe.

milliamps. The Delrin probe has three longitudinal, anteriorly placed, stainless steel, bar electrodes. The probe is blunt at the tip and a thermocouple in the middle electrode monitors temperature at the electrode-mucosal interface. The temperature is shown on an LED screen built into the stimulator.

Proctoscopy was performed to determine the health of the rectal mucosa before the rectal probe was inserted. The stimulator output was progressively increased to the maximum voltage $(16 \mathrm{~V})$ or until ejaculation occurred. Stimulation ceased if the temperature reached $40{ }^{\circ} \mathrm{C}$. Emission of semen occurred through the urethra around the catheter. Semen was collected in a warm sterile plastic jar. Proctoscopy was repeated after the procedure and the catheter was removed.

To determine the adequacy of bladder neck tamponade, urine was collected following the procedure. This practice was discontinued after the first three EEs on each patient, as there was no sperm in the urine.

\section{Vibration ejaculation}

After catheterisation (as above), ejaculation was induced with a commercially available domestic vibrator (Jeou Jen Electric Co) at $60 \mathrm{Hertz}$. The procedure was similar to that described by Brindley. ${ }^{1}$

\section{Semen analysis}

The ejaculate volume was measured in a graduated syringe after liquefaction. Sperm concentration was measured in a haemocytometer chamber using the appropriate dilution. ${ }^{10}$ Sperm morphology was assessed from smears stained using the Shorr procedure, with 200 sperm being graded according to criteria previously described. ${ }^{11}$ The percentage of motile sperm was assessed by the method described below.

\section{Effect of catheters and lubricants on sperm Patients}

Samples were provided by five men being investigated for infertility. Specimens were collected at the laboratory by masturbation into a sterile plastic container after a recommended minimum period of abstinence of 3 days. Normal and abnormal specimens were selected for this study to cover the spectrum of sperm quality in patients with SCI.

\section{Catheters and lubricants}

Three compositions of Foley '14 French gauge-5cc' catheters were selected for study: All latex (Bardex ${ }^{\circledR}$ : C.R. Bard Inc), silicone-coated latex (Bardia ${ }^{\circledR}$ : C.R Bard Inc.) 
and all silicone (Argyle ${ }^{\circledR}$ : Sherwood Medical; Bardex ${ }^{\circledR}$ : C.R Bard Inc.; Curity ${ }^{\circledR}$ : The Kendall Co. and 4 Sure ${ }^{\circledR}$ : Boston \& Pacific Co). The lubricants chosen were the most widely used preparations: Petroleum jelly (Vaseline ${ }^{\circledR}$, Chesebrough Ponds International Ltd), K-Y ${ }^{\circledR}$ jelly (Johnson \& Johnson Ltd), Surgilube ${ }^{\circledR}$ (Fougera \& Co).

A $1 \mathrm{~mm}$ thick cross section of each catheter was placed into separate sterile plastic tubes. Using a sterile spatula a small quantity of each lubricant was spread onto the bottom of sterile plastic test tubes to form a thin coating. A $0.2 \mathrm{ml}$ aliquot of semen was added to each tube. The tubes were placed into an incubator $\left(35^{\circ} \mathrm{C}\right)$ fitted with a moving tray which gently mixed the semen for 30 minutes. Positive and negative controls were repectively: $1 \mathrm{~mm}$ sections of latex glove which is known to completely immobilise sperm, ${ }^{12}$ and a tube containing only semen.

\section{Motility analysis \\ Computer assessment}

The Hamilton-Thorn Motility Analyser was used to assess the motility characteristics of each sample. The procedure involves the placing of a $5 \mu \mathrm{l}$ aliquot of semen into a $10 \mu$ m-deep Makler chamber at $37^{\circ} \mathrm{C}$ and loading the chamber into the instrument. The following were measured: average path speed (VAP in $\mu \mathrm{m} / \mathrm{s}$ ), mean curvilinear speed (VCL), mean straight line speed (VSL), mean linearity (LIN), mean straightness (STR), mean amplitude of lateral head displacement (ALH) and mean beat cross frequency $(\mathrm{BCF})$. Also measured were the percentages of motile sperm with VAP in different ranges: total progressive (VAP $>10 \mu \mathrm{m} / \mathrm{s}$ ) and those with rapid (VAP $>30 \mu \mathrm{m} / \mathrm{s})$, medium $(10<\mathrm{VAP}<$ $30)$, slow $(0<\mathrm{VAP}<10)$, static $(\mathrm{VAP}=0)$ speed. The analyses were performed at the settings previously described. ${ }^{13}$ For each specimen an average of nine fields (range 4-18) having a mean number of 354 spermatozoa (range 105-804) was studied.

\section{Manual assessment}

The percentage of motile sperm was determined by microscopic examination $(\times 400)$ of $7 \mu \mathrm{l}$ of semen placed on a warm $\left(37^{\circ} \mathrm{C}\right)$ glass slide and covered with a glass coverslip $(22 \mathrm{~mm} \times 22 \mathrm{~mm})$. At least 200 sperm were counted and graded according to the $\mathrm{WHO}^{10}$ criteria. Nonmotile sperm were assigned a score of zero; motile sperm were graded as: 1 (no forward progression), 2 (forward progression) and 3 (rapid, linear, forward progression). Progression was assessed subjectively with fast forward progression being defined as rapid, straight line movement. The motility index ${ }^{14}$ was calculated by adding the product of the grade and the percentage of sperm in that grade. Semen samples without specific motility disorders have a motility index (MI) of about twice the percentage motility. The manual percentage motility is higher than the computer measuerement because the computer only counts as motile those sperm with a VAP $>10 \mu \mathrm{m} / \mathrm{s}$.

\section{Sperm viability $(S V)$}

The percentage of dead sperm was determined using the eosin $Y$ wet method, ${ }^{15}$ which is based on the principle that dead sperm with damaged plasma membranes take up stain.

\section{Statistical analysis}

The data were compared by two way analysis of variance (ANOVA) and comparison of differences of the means for the control and experimental samples using the least significant difference method. Calculations were performed using the Statistical Package for Interactive Data Analysis (Statistical Computing laboratory, Macquarie University, Australia).

\section{Results}

\section{Assisted ejaculation}

Over a period of 26 months, a total of 100 procedures (84 EE and $16 \mathrm{VE}$ ) were performed, with each patient undergoing between one and 23 assisted ejaculations. Both $\mathrm{VE}$ and EE procedures were usually completed within 20 minutes and resulted in antegrade ejaculations being collected from 
all patients. No incidences of severe hypertension due to autonomic dysreflexia or visually apparent urine contamination of the semen were noted. Post EE proctoscopy showed no damage to the rectal mucosa of any patient.

A wide variation in semen quality was found both between patients and in samples collected from the same patient (Table II). In $92 \%$ of cases, the first sample produced at least three semen characteristics below the recommended normal range ${ }^{10}: 92 \%$ of first samples had either semen volumes less than $2 \mathrm{ml}$ or less than $30 \%$ of sperm with normal morphology or less than $40 \%$ motile sperm. $59 \%$ had sperm concentrations below 20 million per ml. Subsequent samples showed a marked improvement in semen quality with $60 \%$ of patients producing specimens with increased sperm concentrations and $50 \%$ having either semen volume, percentage motile sperm or percentage of sperm with normal forms exceeding the lower limit of the normal range. The greatest differences seen were in sperm motility, where the first sample of 10 of the 12 patients contained less than $10 \%$ motile sperm, seven having no motile sperm. Subsequent samples from nine of the 10 patients who underwent the procedure on a number occasions, were found to have sperm motilities greater than $30 \%$. The one patient (number 4, Table II) who showed no improvement had severe hypospermatogenesis on testicular biopsy. Two patients (numbers 3 and 4) underwent EE after successful VE, due to fears that vibratory stimulation might induce uncontrollable autonomic dysreflexia. Ejaculate volumes and sperm concentrations collected by $\mathrm{EE}$ were greater than those obtained by VE. No difference in sperm motility was found between the two methods.

\section{Complications}

One patient had significant problems with recurrent urinary tract infections (enterococcus faecalis) which were detected in post EE urine samples. The only other difficulty encountered was a patient with incomplete low level paraplegia who complained of severe stomach cramps during the EE procedure. As even low voltages caused discomfort, subsequent EEs were performed under general anaesthesia and have resulted in the collection of satisfactory specimens.

\section{Effect of catheters and lubricants on sperm} All catheters except 4 Sure $^{\circledR}$ significantly decreased the manually assessed motility and MI below those for the control specimens (Table III). The all latex $\left(\right.$ Bardex $\left.^{\circledR}\right)$

Table II Range of semen characteristics for each patient

\begin{tabular}{|c|c|c|c|c|c|c|}
\hline Subjects & $\begin{array}{c}\text { No. of } \\
\text { Specimens }\end{array}$ & $\begin{array}{l}\text { Volume } \\
\text { (ml) }\end{array}$ & $\begin{array}{c}\text { Concentration } \\
\left(10^{6} / \mathrm{ml}\right)\end{array}$ & $\begin{array}{l}\text { Motility } \\
(\%)\end{array}$ & $\begin{array}{c}\text { Progress motility } \\
\qquad(\%)\end{array}$ & $\begin{array}{l}\text { Normal forms } \\
(\%)\end{array}$ \\
\hline 1 & 1 & 5.5 & 82 & 1 & ND" & 10 \\
\hline 2 & 1 & 0.4 & 9 & 4 & 4 & 14 \\
\hline 3 & 5 & $0.5-1$ & $500-1970$ & $10-32$ & $8-21$ & $21-23$ \\
\hline 4 & 4 & $1.2-1.9$ & $(0-2$ & $0-10$ & $(0-8$ & 10 \\
\hline 5 & 23 & $0.5-4.0$ & $2-576$ & $0-80$ & $0-64$ & $11-46$ \\
\hline 6 & 15 & $0.1-4.0$ & $1-236$ & $4-55$ & $3-40$ & $6-20$ \\
\hline 7 & 17 & $0.1-2.8$ & $0-4$ & $0-45$ & $0-36$ & $1-40$ \\
\hline 8 & 11 & $0.2-3.5$ & $286-965$ & $(0-30$ & $(0-24$ & $5-30$ \\
\hline 9 & 13 & $1-8.5$ & $1-54$ & $(0-40$ & $0-38$ & $6-19$ \\
\hline 10 & 2 & $0.5-1.2$ & $305-500$ & $(0-50$ & $(0-4$ & $10-15$ \\
\hline 11 & 4 & $0.1-0.2$ & $42-400$ & $(0-30$ & $0-24$ & $10-20$ \\
\hline 12 & 4 & $0.5-1.4$ & $0-8$ & $(0-50$ & ND & $20-30$ \\
\hline $\begin{array}{l}\text { Normal } \\
\text { range }\end{array}$ & & $>2.0$ & $>20$ & $>40$ & $>25$ & $>30$ \\
\hline
\end{tabular}

a ND $=$ not done. 


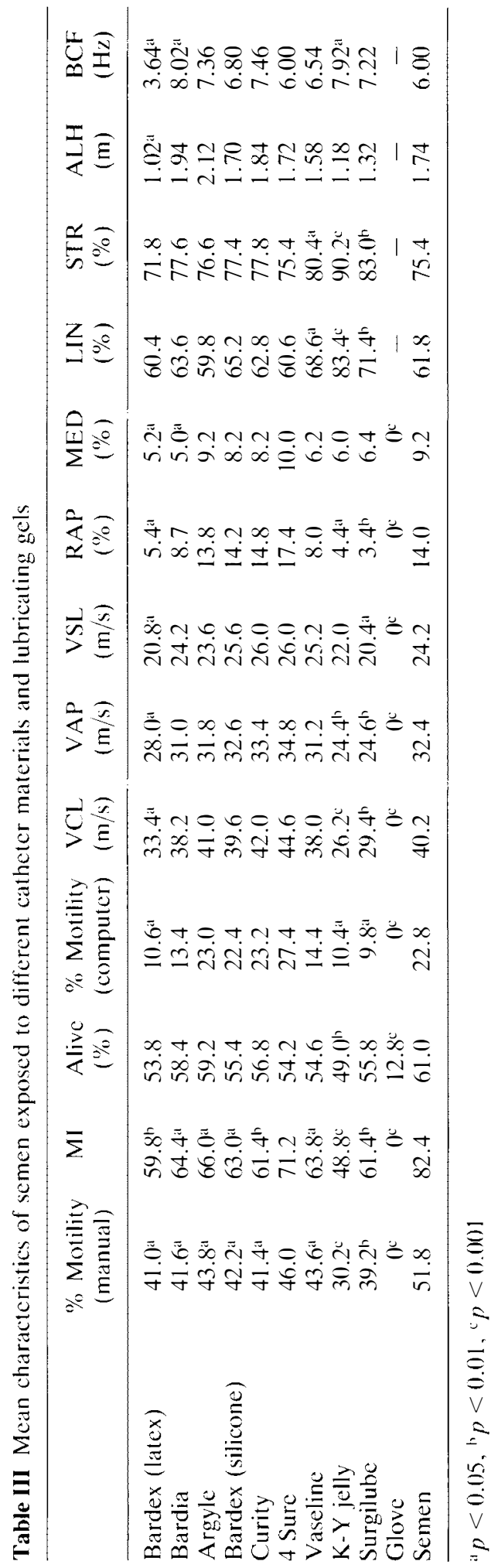

catheter was also found to significantly lower the computer assessed motility measurements-VAP, VCL, VSL, ALH and $\mathrm{BCF}$. The only other difference noted was that the silicone coated latex (Bardia ${ }^{\circledR}$ ) catheter significantly decreased the percentage of sperm with medium speed but increased the BCF. SV was not affected significantly by any of the catheters.

All the lubricants tested were found to decrease manually assessed motility and MI, but to increase LIN and STR. Sperm placed in the aqueous gels (K-Y jelly ${ }^{\circledR}$ and Surgilube $\left.{ }^{\circledR}\right)$ were also found to have significantly lower computer asessed total progressive motility, VAP, rapid speed and VCL than for the control specimen. In addition, K-Y jelly ${ }^{\circledR}$ caused a significant decrease of SV and increase of BCF. Surgilube ${ }^{\circledR}$ also significantly decreased VSL.

\section{Discussion}

Male infertility due to ejaculatory dysfunction is a major problem associated with chronic SCI. Although assisted ejaculation techniques, such as $\mathrm{EE}$ and $\mathrm{VE}$, have provided a means of collecting semen from men with SCI, partial or complete retrograde ejaculation is common. To prevent the detrimental effects of urine on already compromised semen and to circumvent the complicated procedures involved in sperm retrieval from the bladder, we used the simple technique of balloon catheter bladder neck tamponade.

The addition of this technique to our assisted ejaculation protocols resulted in the collection of antegrade ejaculations from all patients. The lack of urine contamination and the absence of sperm from post ejaculatory urine, indicated that the catheter had successfully tamponaded the bladder neck. The procedure caused minimal inconvenience to the patients, most being accustomed to catheterisation as part of their bladder training. No serious complications were encountered with the procedures, although early in the study one patient had recurrent urinary tract infections following EE.

All but one of the catheters tested were found to significantly decrease manually 
assessed motility and MI. The finding that the 4 Sure $^{\circledR}$ catheter did not cause any changes to the sperm motility, suggests that the damage caused by the other three $100 \%$ silicone catheters, may not be due to the silicone base but rather to other factors such as coatings applied to the outside of the catheters. Considering the dramatic effect of the latex glove on sperm movement and viability, the finding that the all-latex catheter affected almost every aspect of sperm motility was not surprising. The application of a silicone coating appeared to reduce this problem. The all silicone catheters were selected for use in our procedure because they caused the least reduction in sperm motility and were easily inserted.

The use of lubricants is a common element in catheterisation. However, for semen collection, it is recommend that lubricating gels (especially petroleum based) are avoided because of their deleterious effect on sperm survival and motility. ${ }^{9,10}$ These studies showed that the petroleum based gel (Vaseline ${ }^{\circledR}$ ) decreased only the manually assessed motility. Yet the aqueous based lubricants, which are most often recommended by infertility clinics, adversely affected almost all the sperm characteristics measured. All the lubricants tested were found to significantly increase mean linearity and mean straightness, a not unexpected result as the viscosity of the gels would cause the sperm to swim in a more linear fashion with a higher beat cross frequency to maintain forward progression. But only K-Y jelly ${ }^{\circledR}$ showed a significant difference from the control specimen. Processing semen samples which have come into contact with lubricants may be difficult because the gels concentrate in the centrifuged sperm pellet. Thus we decided to avoid the use of conventional lubricants.
The quality and quantity of the initial semen samples collected from our patients were comparable to those of other studies: low volumes, variable sperm concentrations and poor sperm motility. Our observations agree with the studies of Brindley ${ }^{1}$ who reported that in chronic SCI patients, the first sample collected was generally poor but with successive ejaculations there was a tendency for the quality to improve. These variations in semen quality probably occur because of the inability of chronic SCI patients to ejaculate. When assisted ejaculation procedures are performed on these patients, the first sample collected usually contains large numbers of dead and degenerating sperm which have accumulated in the genital tract over time. Successive ejaculations empty the reserves of aged sperm allowing the epididymis to be replenished by young, viable sperm with increased motility.

\section{Conclusions}

Tamponading the bladder neck by balloon catheter was found to be an easy, quick and safe method of preventing retrograde ejaculation during assisted ejaculation procedures. Conventional lubricants for catheterisation should be avoided because they impair sperm motility and make processing sperm difficult. Silicone catheters should be chosen for the procedure because of their minimal effect on sperm and ease of use.

\section{Acknowledgements}

We would like to thank Peter Elliot and Gary Clarke of the Andrology Laboratory, Royal Women's Hospital for their help in the catheter and lubricant study.

\section{References}

1 Brindley GS (1984) The fertility of men with spinal injuries. Paraplegia 22: 337-348.

2 Halstead LS, VerVoort S, Seager SW (1987) Rectal probe electrostimulation in the treatment of anejaculatory spinal cord injured men. Paraplegia 25: 120-129.

3 Siosteen A, Forssman L, Steen Y, Sullivan L, Wickstrom I (1990) Quality of semen after repeated ejaculation treatment in spinal cord injured men. Paraplegia 28: 96-104.

4 Linsenmeyer TA, Perkash I (1991) Infertility in men with spinal cord injury. Arch Phys Med Rehabil 72: 747-754

5 Hershlag A, Schill SF, DeCherney AH (1991) Retrograde ejaculation. Hum Reprod 6: 225-258. 
6 Warner H, Martin DK, Perkash I, Speck V, Nathan B (1986) Electrostimulation of erection and ejaculation and collection of semen in spinal cord injured humans. J Rehabil Res Dev 23: 21-31.

7 Perkash I, Martin DE, Warner H, Speck V (1990) Electroejaculation in spinal cord injured patients: simplified new equipment and technique. J Urol 143: 305-7.

8 Rawicki B, Hill S (1991) Semen retrieval in spinal cord injured men. Paraplegia 29: 443-446.

9 Linsenmeyer T, Wilmont C, Anderson RU (1989) The effects of the electroejaculation procedure on sperm motility. Paraplegia 27: 465-469.

10 World Health Organisation (1992) Laboratory Manual for the Examination of Human Semen and Sperm-Cervical Mucus Interaction. Cambridge University Press, Cambridge.

11 Liu DY, Baker HWG (1992) Tests of sperm function and fertilisation in vitro. Fertil Steril 58: 465-483.

12 Jones DM, Kovacs GT, Harrison L, Jennings MG, Baker HWG (1986) Immobilisation of sperm by condoms and their components. Clin Reprod Fertil 4: 367-372.

13 Liu DY, Clarke GN, Baker HWG (1991) Relationship between sperm motility assessed with the Hamilton-Thorn motility analyser and fertilisation rates in virto. J Androl 12: 231-239.

14 MacLeod J (1969) Further observations on the role of varicocele in human male infertility. Fertil Steril 20: 545-563.

15 Eliasson R (1977) Supravital staining of human spermatozoa Fertil steril 28: 1257. 